\title{
hBMP-7 induces the differentiation of adipose-derived mesenchymal stem cells into osteoblast-like cells
}

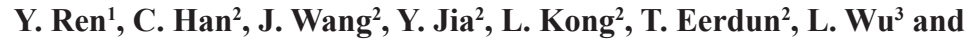 \\ D. Jiang ${ }^{1}$
}

${ }^{1}$ Department of Orthopedics, The First Hospital of Chongqing Medical University, Yuzhong District, Chongqing, China

${ }^{2}$ Department of Arthroscopy and Sports Medicine,

The Second Affiliated Hospital of Inner Mongolia Medical University,

Hyumin District, Huhehot, Inner Mongolia, China

${ }^{3}$ Department of Anesthesiology,

The Second Hospital of Inner Mongolia Medical University,

Huhehot, Inner Mongolia, China

Corresponding author: D. Jiang

E-mail: Jdm571026@vip.163.com

Genet. Mol. Res. 15 (3): gmr.15038791

Received May 16, 2016

Accepted June 3, 2016

Published July 25, 2016

DOI http://dx.doi.org/10.4238/gmr.15038791

Copyright $(2016$ The Authors. This is an open-access article distributed under the terms of the Creative Commons Attribution ShareAlike (CC BY-SA) 4.0 License.

ABSTRACT. The aim of this study was to investigate the differentiation
potential of adipose-derived mesenchymal stem cells (ADMSCs)
into osteoblasts by human bone morphogenetic protein-7 (hBMP-7)
induction. ADMSCs were isolated from the subcutaneous adipose tissue
of a rabbit, and then transfected with the pcDNA3.1 vector alone and
pcDNA3.1-hBMP-7 (hBMP-7), respectively. Untransfected ADMSCs
were used as the control group. After transfection, the morphology and
green fluorescent protein (GFP) fluorescence intensity of ADMSCs

Genetics and Molecular Research 15 (3): gmr.15038791 
were observed by fluorescent microscopy. The 3-(4,5-dimethylthiazol2-yl)-2,5-diphenyltetrazolium bromide assay was performed to detect the growth of ADMSCs at 1, 3, and 5 days, respectively. Transmission electron microscopy was performed to observe the ultrastructural morphology of ADMSCs. In addition, ADMSCs were stained with quinalizarin and toluidine blue to reflect the content of osteoblasts and chondrocytes, respectively. Finally, the expression of collagen I and osteocalcin in ADMSCs was detected by western blot. ADMSCs were successfully isolated. Obvious GFP fluorescence and high expression of hBMP-7 demonstrated the successful transfection of hBMP-7. Specific morphological characters with a metabolically active ultrastructure were exhibited on the ADMSCs transfected with hBMP7. In addition, the growth rate of ADMSCs transfected with hBMP-7 was significantly higher than that of the cells in the vector and control groups. Successfully induced osteoblast-like cells were identified by an obvious erythrine area and high expression of collagen I and osteocalcin in ADMSCs transfected with hBMP-7. Thus, ADMSCs can be successfully differentiated into osteoblast-like cells by hBMP-7 induction in vitro.

Key words: Adipose-derived mesenchymal stem cells; Osteoblasts; hBMP-7; Differentiation; Collagen I

\section{INTRODUCTION}

Mesenchymal stem cells (MSCs) are multipotent stromal cells that exhibit a high capacity of self-renewal and maintenance of multipotency. MSCs can differentiate into multiple cell types (Beyer Nardi and da Silva Meirelles, 2006), including cartilage (Bernardo et al., 2007), muscle (Yoon et al., 2005), bone (Holtorf et al., 2005), and adipose tissue. Growth factors and cytokines can accelerate the rate of cell differentiation (Potapova et al., 2007). In addition, due to the relative ease in establishing in vitro cultures and achieving good proliferation, MSCs have been developed as an attractive model cell to study differentiation mechanisms (Bianco et al., 2001).

Bone regeneration in calvarial defects remains a challenge for surgeons. Along with the substantial progress in effective tissue engineering methods performed on bone regeneration, the application of stem cells has increased (Grayson et al., 2015). MSCs have been shown to differentiate into osteoblasts, and this property has been applied for promoting the bone regeneration of calvarial defects (Koob et al., 2011). In addition, MSCs originating from the local bone marrow, periosteum, endosteum, mineralized bone, or systemic circulation have been shown to play important roles in osteoblastic differentiation and the secretion of factors during the spontaneous healing of a bone trauma or defect (Wang et al., 2013). Adiposederived mesenchymal stem cells (ADMSCs) can also differentiate into various kinds of cells, including adipogenic, osteogenic, chondrogenic, myogenic lineages, and osteoblasts (Mizuno et al., 2002; Zuk et al., 2002; Semyari et al., 2016). The growth factor bone morphogenetic protein (BMP) was originally discovered owing to its ability to induce the formation of bone and cartilage (Sánchez-Duffhues et al., 2015). BMPs have been reported to play important

Genetics and Molecular Research 15 (3): gmr.15038791 
roles in early development and bone inductive activity, which could orchestrate the tissue architecture throughout the body via a group of pivotal morphogenetic signals (Bleuming et al., 2007). However, studies on the induction of ADMSCs into osteoblasts by BMPs are still limited.

In this study, full-length human BMP-7 (hBMP-7) was transfected into ADMSCs, and the growth state and morphological changes of the ADMSCs were observed. In addition, the proliferation of ADMSCs was measured, and the effect of hBMP-7 on osteoblasts differentiation was evaluated. Our findings may reveal the process of the hBMP-7-induced differentiation of ADMSCs into osteoblasts, and could provide a new perspective for bone regeneration in a clinical setting.

\section{MATERIAL AND METHODS}

\section{Isolation and culture of ADMSCs}

Subcutaneous adipose tissues of about $5 \mathrm{~cm}^{3}$ were obtained from the nape of the neck of a male New Zealand rabbit (weighing 2.5-3.0 kg) provided by the Animal Experimental Center of China Medical University, Shenyang, Liaoning, China. The obtained tissue was washed with phosphate buffered saline, and cut into small pieces of 1-3 $\mathrm{mm}^{3}$. After digestion with $0.1 \%$ type-I collagenase (Invitrogen, Carlsbad, CA, USA) at $37^{\circ} \mathrm{C}$ for $1 \mathrm{~h}$, the precipitate was collected by centrifugation at $400 \mathrm{~g}$ for $10 \mathrm{~min}$ and was re-suspended by $\mathrm{NH}_{4} \mathrm{Cl}(0.16 \mathrm{M})$ at room temperature for $15 \mathrm{~min}$ to hemolyze the red blood cells. The solution was then filtered through a 200-mm filter, and cultured in Dulbecco's modified Eagle medium with $10 \mathrm{mM}$ glucose and $5 \%$ fetal bovine serum at $37^{\circ} \mathrm{C}$. After culturing for $48 \mathrm{~h}$, the culture medium was initially replaced daily until the cells grew to fill the bottom of the container. Then, the cells were digested with $0.25 \%$ pancreatic enzyme and $0.02 \%$ ethylenediaminetetraacetic acid, and finally generated, using a ratio of 1:2. The study was approved by the Experimental Animal Ethics Committee at China Medical University.

\section{Transfection of hBMP-7}

The encoding sequence of hBMP-7 cDNA $(1.3 \mathrm{~kb})$ was amplified from pBluescripthBMP-7 (provided by Dr. Yu Bing, Fourth Military Medical University, Shanxi, China). The segment was connected with the pcDNA3.1 plasmid vector (Invitrogen), and transfected into Escherichia coli DH5 $\alpha$. After accurate sequencing, the recombinant plasmid pcDNA3.1hBMP-7 (hBMP-7) was extracted using a large-quantity plasmid extraction kit (Omega Biotek, Inc., Doraville, GA, USA).

The second generation of ADMSCs was seeded at a density of $0.5 \times 10^{6}$ cells/well in 6-well plates, and then transfected with pcDNA3.1 (vector) or hBMP-7 using Lipofectamine 2000, according to the manufacturer instructions (Invitrogen). Untransfected ADMSCs were used as the control group. At $24 \mathrm{~h}$ post-transfection, the cells were inoculated onto fresh culture lysates and diluted at 1:20 for passage 2 . After $48 \mathrm{~h}$, the transfected cells were cultured for 14 days, and geneticin (G418, Gibco-BRL, $500 \mathrm{mg} / \mathrm{mL}$ ) was used to select geneticin-resistant transfected cells. The green fluorescent protein (GFP) fluorescence intensity and morphology of ADMSCs in the different groups were observed on a BX50 fluorescence microscope (Olympus, Tokyo, Japan).

Genetics and Molecular Research 15 (3): gmr.15038791 


\section{Reverse transcription-polymerase chain reaction (PCR)}

Total RNA from the ADMSCs of each group was isolated by TRIzol reagent (Invitrogen). RevertAid First-Strand cDNA Synthesis Kit (Fermentas, Hanover, MD, USA) was used for reverse transcription. The specific primers for reverse transcription-PCR were as follows: hBMP-7 forward, 5'-GGT CTA GAA TGC ACG TGC GCT CAC TGCG-3'; hBMP7 reverse, 5'-CC GGT ACC TTA GTG GCA GCC ACA GGC CCGG-3'. $\beta$-actin was used as an internal control. The sequences of the $\beta$-actin primers were 5 '-CTC CTT AAT GTC ACG CAG GAT TTC-3' (forward primer) and 5'-GTG GGG CGC CCC AGG CAC CA-3' (reverse primer). The PCR program included $94^{\circ} \mathrm{C}$ for $5 \mathrm{~min}$, then 30 cycles of $94^{\circ} \mathrm{C}$ for $1 \mathrm{~min}, 56^{\circ} \mathrm{C}$ for $1 \mathrm{~min}$, and $72^{\circ} \mathrm{C}$ for $2 \mathrm{~min}$, with a final extension at $72^{\circ} \mathrm{C}$ for $2 \mathrm{~min}$. PCR products were resolved and documented by $1 \%$ agarose gel electrophoresis.

\section{3-(4,5, Dimethylthiazol-2-yl)-2,5-diphenyltetrazolium bromide (MTT) assay}

The proliferation of ADMSCs in the different groups was detected by an MTT assay following the manufacturer instructions (Amresco, Solon, OH, USA) at 1, 3, and 5 days, respectively. In brief, $250 \mathrm{mg}$ MTT was added to the ADMSCs at $0.5 \times 10^{5}$ cells/well in 96-well plates, and the optical density was detected at $570 \mathrm{~nm}$ using an ultraviolet spectrophotometer (Bio-Rad, Hercules, CA, USA).

\section{Quinalizarin and toluidine blue staining, and transmission electron microscopy (TEM)}

After transfection, quinalizarin and toluidine blue staining were performed on the ADMSCs of different groups and observed using a fluorescent microscope (Olympus) to determine the contents of osteoblasts and chondrocytes. In addition, the ultrastructural morphology of ADMSCs in the different groups was observed using TEM (Philips CM 120, Eindhoven, Netherlands). After the cells were fixed, they were dehydrated by acetone, soaked in propylene oxide at $24^{\circ} \mathrm{C}$ for $24 \mathrm{~h}$, and embedded in araldite. Subsequently, the embedded specimens were cut into ultrathin slices, stained with lead citrate, and examined under TEM.

\section{Western blot}

After isolation of the cellular lysates from ADMSCs, the proteins were separated by sodium dodecyl sulfate-polyacrylamide gel electrophoresis on $10 \%$ polyacrylamide gels and transferred to a polyvinylidene fluoride membrane. The membrane was blocked with $5 \%$ skim milk in Tris-buffered saline containing $0.05 \%$ Tween- 20 for $2 \mathrm{~h}$, and then specific diluted primary antibodies against collagen I and osteocalcin (1:100, Neumark, USA) were added. The membrane was then incubated overnight at $4^{\circ} \mathrm{C}$, washed in Tris-buffered saline with Tween-20 three times, and incubated with fluorescein isothiocyanate-conjugated goat antimouse antibody (Zhongshan Jinqiao, Beijing, China) for $1 \mathrm{~h}$ at $25^{\circ} \mathrm{C}$. Finally, after the samples were washed, the gel image was analyzed semi-quantitatively using an ultraviolet gel imager (Shanghai Tianneng Co., Ltd., Shanghai, China).

\section{Statistical analyses}

All data are expressed as means \pm standard deviations. Comparison between different

Genetics and Molecular Research 15 (3): gmr.15038791 
groups was determined with the Student t-test. A P value $<0.05$ was considered to indicate a significant difference. Statistical analysis was performed on SPSS version 12.0 (SPSS Inc., Chicago, IL, USA).

\section{RESULTS}

\section{Morphology of ADMSCs}

No GFP fluorescence of ADMSCs was found in the control group (Figure 1A) or vector group (Figure 1B). However, obvious GFP fluorescence was observed in the hBMP-7 group, which indicated the successful transfection of hBMP-7 into ADMSCs (Figure 1C). After $48 \mathrm{~h}$ of culture, the ADMSCs were considered to be successfully isolated, and those of the control (Figure 1D) or vector group (Figure 1E) exhibited a typical elliptical or fibroblastic morphology. Furthermore, specific morphological characters, including numerous mitotic figures, an increased cell volume with polygonal, oval, or irregular shape, a large and oval nucleus with a prominent nucleolus, and emerged vacuoles in the cytoplasm, were exhibited on the cells in the hBMP7 group (Figure 1F). hBMP-7 was expressed in the hBMP-7 group of ADMSCs, whereas no expression of hBMP-7 was found in the vector or control group (Figure 2).

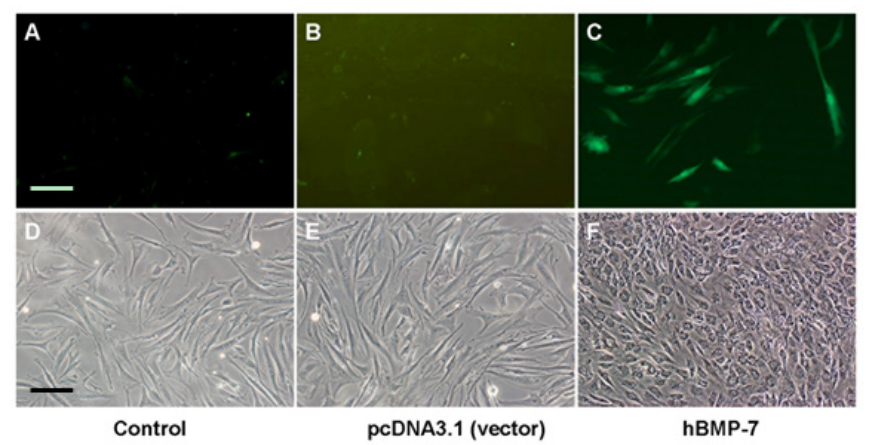

Figure 1. Green fluorescent protein (GFP) fluorescence and morphology observation (200X) of adipose-derived mesenchymal stem cells (ADMSCs) transfected in the control group (A, D), vector group (B, E) and hBMP-7 group (C, F). Scale bar, $200 \mathrm{~mm}$.

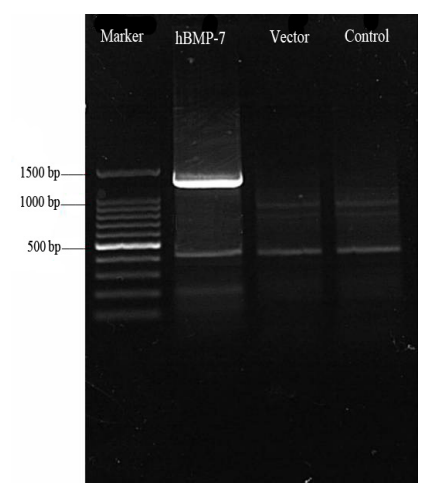

Figure 2. Expression of hBMP-7 in ADMSCs in different groups was examined by RT-PCR.

Genetics and Molecular Research 15 (3): gmr.15038791 


\section{The growth of ADMSCs was improved by hBMP-7}

The proliferation evaluation of ADMSCs using MTT assays revealed that the ADMSCs transfected with hBMP-7 exhibited rapid growth, and the growth rate was higher than that of the vector and control groups at 1, 3, and 5 days (Figure 3). In addition, ADMSCs transfected with the vector showed a similar growth rate to those of the control group.

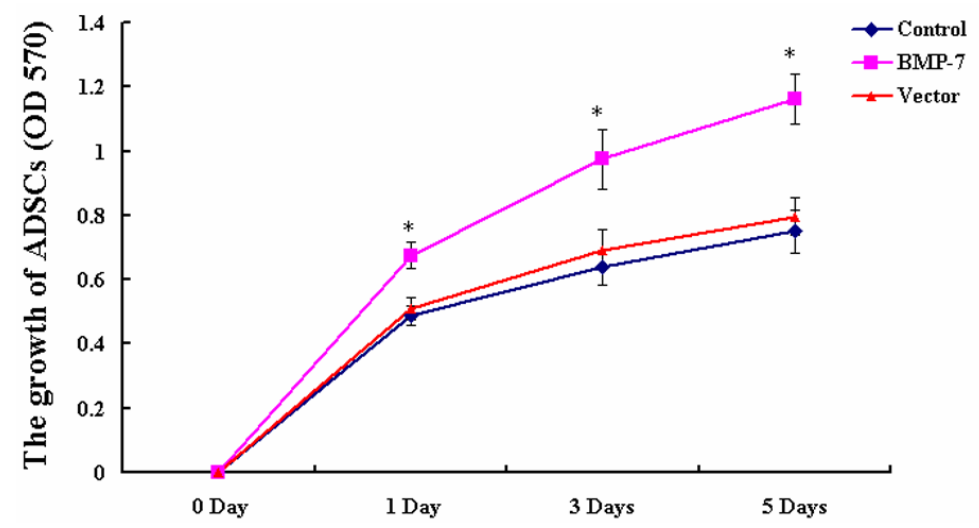

Figure 3. Proliferation of ADMSCs transfected with pcDNA3.1 or hBMP-7. Untransfected ADMSCs were used as control group. ${ }^{*} \mathrm{P}<0.05$ compared with vector and control group.

\section{Ultrastructural observation of ADMSCs transfected with hBMP-7}

The ultrastructural observation of ADMSCs in the vector (Figure 4A) or control group (Figure 4B) demonstrated obvious immature characteristics, including an oval-shaped nucleus with a prominent nucleolus, chromatin with a diffuse and scattered distribution, and few organelles. In addition, the Golgi apparatuses, mitochondria, or endoplasmic reticula were poorly developed in these two groups. By contrast, the ADMSCs in the hBMP-7 group had denser chromatin with plenty of well-developed organelles, which indicated that these cells were metabolically activated (Figure 4C).

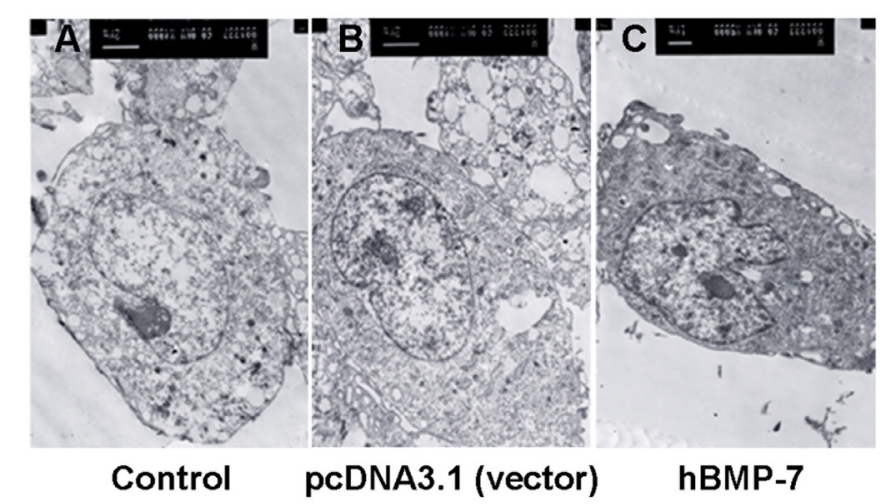

Figure 4. Ultrastructural observation of (A) control group (untransfected ADMSCs); (B) ADMSCs transfected with pcDNA3.1; and (C) ADMSCs transfected with hBMP-7. Original magnification: 7500X.

Genetics and Molecular Research 15 (3): gmr.15038791 


\section{Formation of osteoblast-like cells in ADMSCs transfected with hBMP-7}

After staining with quinalizarin, no erythrine was observed in the control (Figure $5 \mathrm{~A}$ ) and vector groups (Figure 5B). However, an obvious erythrine area was observed in the ADMSCs transfected with hBMP-7 (Figure 5C), which reflected high contents of osteocalcin. Furthermore, we assessed the distribution of chondrocytes in ADMSCs by toluidine blue staining, and found that the number of chondrocytes in the control (Figure 5D) and vector groups (Figure 5E) was lower than that in the hBMP-7 group (Figure 5F).

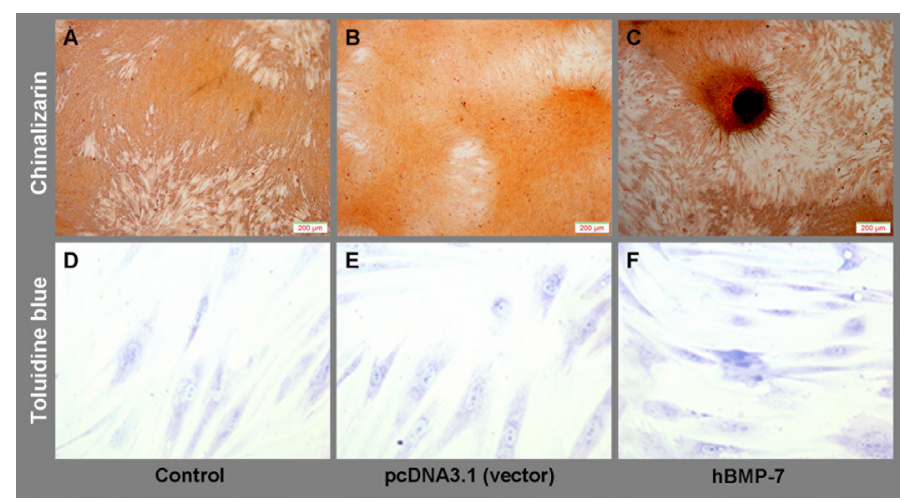

Figure 5. Chinalizarin and toluidine blue staining of (A, D) untransfected adipose-derived mesenchymal stem cells (ADMSCs), (B, E) ADMSCs transfected with pcDNA3.1 and (C, F) ADMSCs transfected with hBMP-7.

\section{hBMP-7 induced high collagen I and osteocalcin expression}

Both collagen I and osteocalcin were expressed in the three different groups of ADMSCs (Figure 6A). In addition, compared with the vector and control groups, the levels of collagen I and osteocalcin in the hBMP-7 group were higher (Figure 6B). The levels of collagen I and osteocalcin in the vector and control groups were relatively low, with no significant difference observed between these two groups (Figure 6B).

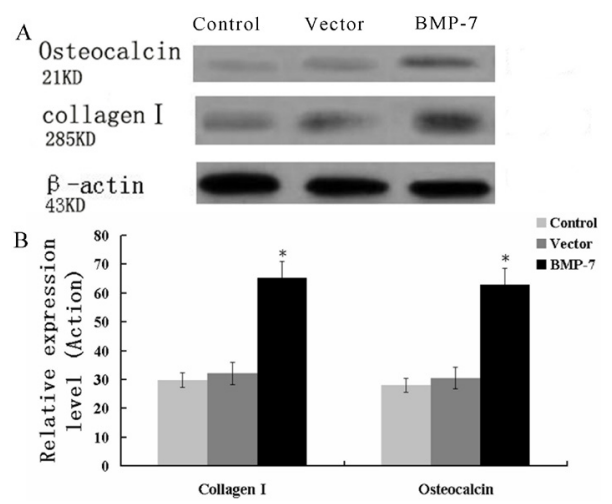

Figure 6. The expression (A) and relative expression levels (B) of collagen I and Osteocalcin in adipose-derived mesenchymal stem cells (ADMSCs) transfected with pcDNA3.1 and hBMP-7. Untransfected ADMSCs were used as control group. ${ }^{*} \mathrm{P}<0.05$ compared with vector and control group.

Genetics and Molecular Research 15 (3): gmr.15038791 


\section{DISCUSSION}

Recently, stem cells have been widely used in tissue-engineering strategies for bone defects and repair (Seong et al., 2010). An applicable cell source with an abundant supply, easy accessibility, and no immunogenic reaction with osteoplastic potential is required for the successful cell-based treatment of bone (Yamada et al., 2013). ADMSCs can differentiate into adipogenic, osteogenic, chondrogenic, myogenic lineages, and osteoblasts (Mizuno et al., 2002; Zuk et al., 2002; Semyari et al., 2016). The technique of ADMSCs isolation from the subcutaneous adipose tissue of rabbits, in vitro culture, and expansion has been previously reported (An et al., 2010). In the current study, we evaluated the effect of hBMP-7 on ADMSCs differentiation to generate osteoblast-like cells, and found that ADMSCs transfected with hBMP-7 exhibited robust proliferation and were capable of producing osteoblast-like cells.

BMPs are closely associated with cell growth and bone formation (Kung and Ries, 2007). We have also demonstrated the prominent growth rate of ADMSCs transfected with hBMP-7 in comparison with the vector or control, similar to previous descriptions of adipose stem cells (Knippenberg et al., 2006). As a specific osteoblastic growth factor in the activation of the bone repair process, BMP-7 is also the initiating signal molecule for the osteoblastic differentiation of MSCs. The stimulation effects of hBMP-7 in osteoblastic differentiation and its effectiveness in bone repair have been verified (Boon et al., 2011). As reported, BMP-7 is beneficial for the attachment, proliferation, and differentiation of pre-osteoblasts, and thus plays important roles in bone tissue regeneration (Mantripragada and Jayasuriya, 2014). In addition, ADMSCs have the ability to form osteoblasts and maintain a differentiated osteoblast phenotype for a long time in vitro (Lu et al., 2008).

In this study, hBMP-7 was successfully transfected into ADMSCs. In addition, specific morphology, obvious quinalizarin staining, a significantly increased growth rate, and higher collagen I and osteocalcin expression in the hBMP-7 group suggested the formation of osteoblast-like cells. During this process, transfected ADMSCs may react with cell membrane receptors via hBMP-7, thereby inhibiting adipose cell differentiation and inducing osteoblastic differentiation (Liu et al., 1999).

Moreover, ADMSCs show chondrogenic potential, with the ability to maintain the differentiated chondrocyte phenotype for a long time both in vivo and in vitro (Erickson et al., 2002), and exhibit a strong capacity to form chondrocytes (Kessler et al., 2008). In the current study, the number of chondrocytes in the control and vector groups was lower than that in the hBMP-7 group, suggesting that the differentiation of ADMSCs induced by BMP-7 toward chondrocytes was effective. However, in another study, ADMSCs were shown to exhibit poor chondrogenic capability, which was attributed to the presence of preadipocytes (Liu et al., 2013). Thus, more studies are needed to verify the induction potential of hBMP-7.

In cell regeneration, ADMSCs are capable of multilineage differentiation, and show several advantages such as their abundant supply, high yield, easy accessibility, and non-immunogenic property (Romagnoli and Brandi, 2014). However, their clinical application remains limited. For example, it is unclear how the plasmid replicates and breaks apart in ADMSCs, and whether the induced osteoblasts possess the same biological abilities in the body. Therefore, further research on ADMSCs induction and its potential application in vivo are still needed.

In conclusion, the transfection of hBMP-7 could successfully induce the differentiation of ADMSCs toward osteoblasts with high proliferation capability. ADMSCs may be used as an abundant and effective source for bone regeneration.

Genetics and Molecular Research 15 (3): gmr.15038791 


\section{ACKONWLEDGMENTS}

Research supported by The Inner Mongolia autonomous region natural funded projects (\#2013MS1194) and the National Natural Science Foundation of China (\#31260230); both project names are "Bionic Building Simulation Structure of Natural Ligament Tissue Engineering and Related Research".

\section{REFERENCES}

An C, Cheng Y, Yuan Q and Li J (2010). IGF-1 and BMP-2 induces differentiation of adipose-derived mesenchymal stem cells into chondrocytes-like cells. Ann. Biomed. Eng. 38: 1647-1654. http://dx.doi.org/10.1007/s10439-009-9892-x

Bernardo ME, Emons JA, Karperien M, Nauta AJ, et al. (2007). Human mesenchymal stem cells derived from bone marrow display a better chondrogenic differentiation compared with other sources. Connect. Tissue Res. 48: 132140. http://dx.doi.org/10.1080/03008200701228464

Beyer Nardi N and da Silva Meirelles L (2006). Mesenchymal stem cells: isolation, in vitro expansion and characterization. Handbook Exp. Pharmacol. 174: 249-282. http://dx.doi.org/10.1007/3-540-31265-X_11

Bianco P, Riminucci M, Gronthos S and Robey PG (2001). Bone marrow stromal stem cells: nature, biology, and potential applications. Stem Cells 19: 180-192. http://dx.doi.org/10.1634/stemcells.19-3-180

Bleuming SA, He XC, Kodach LL, Hardwick JC, et al. (2007). Bone morphogenetic protein signaling suppresses tumorigenesis at gastric epithelial transition zones in mice. Cancer Res. 67: 8149-8155. http://dx.doi.org/10.1158/00085472.CAN-06-4659

Boon MR, van der Horst G, van der Pluijm G, Tamsma JT, et al. (2011). Bone morphogenetic protein 7: a broad-spectrum growth factor with multiple target therapeutic potency. Cytokine Growth Factor Rev. 22: 221-229. http://dx.doi. org/10.1016/j.cytogfr.2011.08.001

Erickson GR, Gimble JM, Franklin DM, Rice HE, et al. (2002). Chondrogenic potential of adipose tissue-derived stromal cells in vitro and in vivo. Biochem. Biophys. Res. Commun. 290: 763-769. http://dx.doi.org/10.1006/bbrc.2001.6270

Grayson WL, Bunnell BA, Martin E, Frazier T, et al. (2015). Stromal cells and stem cells in clinical bone regeneration. Nat. Rev. Endocrinol. 11: 140-150. http://dx.doi.org/10.1038/nrendo.2014.234

Holtorf HL, Jansen JA and Mikos AG (2005). Flow perfusion culture induces the osteoblastic differentiation of marrow stroma cell-scaffold constructs in the absence of dexamethasone. J. Biomed. Mater. Res. A 72A: 326-334. http:// dx.doi.org/10.1002/jbm.a.30251

Kessler MW, Ackerman G, Dines JS and Grande D (2008). Emerging technologies and fourth generation issues in cartilage repair. Sports Med. Arthrosc. Rev. 16: 246-254. http://dx.doi.org/10.1097/JSA.0b013e31818d56b3

Knippenberg M, Helder MN, Zandieh Doulabi B, Wuisman PI, et al. (2006). Osteogenesis versus chondrogenesis by BMP2 and BMP-7 in adipose stem cells. Biochem. Biophys. Res. Commun. 342: 902-908. http://dx.doi.org/10.1016/j. bbrc.2006.02.052

Koob S, Torio-Padron N, Stark GB, Hannig C, et al. (2011). Bone formation and neovascularization mediated by mesenchymal stem cells and endothelial cells in critical-sized calvarial defects. Tissue Eng. Part A 17: 311-321. http://dx.doi.org/10.1089/ten.tea.2010.0338

Kung PL and Ries MD (2007). Effect of femoral head size and abductors on dislocation after revision THA. Clin. Orthop. Relat. Res. 465: 170-174.

Liu P, Oyajobi BO, Russell RG and Scutt A (1999). Regulation of osteogenic differentiation of human bone marrow stromal cells: interaction between transforming growth factor-beta and $1,25(\mathrm{OH})(2)$ vitamin $\mathrm{D}(3)$ In vitro. Calcif. Tissue Int. 65: 173-180. http://dx.doi.org/10.1007/s002239900678

Liu S, Shao Y, Lin Q, Liu H, et al. (2013). 7,8-Dihydroxy coumarin promotes chondrogenic differentiation of adiposederived mesenchymal stem cells. J. Int. Med. Res. 41: 82-96. http://dx.doi.org/10.1177/0300060513476614

Lu J, Bhargav D, Wei AQ and Diwan A (2008). Posterolateral intertransverse spinal fusion possible in osteoporotic rats with BMP-7 in a higher dose delivered on a composite carrier. Spine 33: 242-249. http://dx.doi.org/10.1097/ BRS.0b013e318162451b

Mantripragada VP and Jayasuriya AC (2014). Injectable chitosan microparticles incorporating bone morphogenetic protein-7 for bone tissue regeneration. J. Biomed. Mater. Res. A 102: 4276-4289.

Mizuno H, Zuk PA, Zhu M, Lorenz HP, et al. (2002). Myogenic differentiation by human processed lipoaspirate cells. Plast. Reconstr. Surg. 109: 199-209, discussion 210-211. http://dx.doi.org/10.1097/00006534-200201000-00030

Genetics and Molecular Research 15 (3): gmr.15038791 
Potapova IA, Gaudette GR, Brink PR, Robinson RB, et al. (2007). Mesenchymal stem cells support migration, extracellular matrix invasion, proliferation, and survival of endothelial cells in vitro. Stem Cells 25: 1761-1768. http://dx.doi. org/10.1634/stemcells.2007-0022

Romagnoli C and Brandi ML (2014). Adipose mesenchymal stem cells in the field of bone tissue engineering. World J. Stem Cells 6: 144-152. http://dx.doi.org/10.4252/wjsc.v6.i2.144

Sánchez-Duffhues G, Hiepen C, Knaus P and Ten Dijke P (2015). Bone morphogenetic protein signaling in bone homeostasis. Bone 80: 43-59. http://dx.doi.org/10.1016/j.bone.2015.05.025

Sanchez-Sotelo J, Haidukewych GJ and Boberg CJ (2006). Hospital cost of dislocation after primary total hip arthroplasty. J. Bone Joint Surg. Am. 88: 290-294. http://dx.doi.org/10.2106/JBJS.D.02799

Semyari H Rajipour M, Sabetkish S, Sabetkish N, et al. (2016). Evaluating the bone regeneration in calvarial defect using osteoblasts differentiated from adipose-derived mesenchymal stem cells on three different scaffolds: An animal study. Cell Tissue Bank 17: 69-83.

Seong JM, Kim BC, Park JH, Kwon IK, et al. (2010). Stem cells in bone tissue engineering. Biomed. Mater. 5: 062001. http://dx.doi.org/10.1088/1748-6041/5/6/062001

Wang X, Wang Y, Gou W, Lu Q, et al. (2013). Role of mesenchymal stem cells in bone regeneration and fracture repair: a review. Int. Orthop. 37: 2491-2498.

Yamada Y, Nakamura S, Ito K, Umemura E, et al. (2013). Injectable bone tissue engineering using expanded mesenchymal stem cells. Stem Cells 31: 572-580. http://dx.doi.org/10.1002/stem.1300

Yoon YS, Lee N and Scadova H (2005). Myocardial regeneration with bone-marrow-derived stem cells. Biol. Cell 97: 253-263. http://dx.doi.org/10.1042/BC20040099

Zuk PA, Zhu M, Ashjian P, De Ugarte DA, et al. (2002). Human adipose tissue is a source of multipotent stem cells. Mol. Biol. Cell 13: 4279-4295. http://dx.doi.org/10.1091/mbc.E02-02-0105

Genetics and Molecular Research 15 (3): gmr.15038791 